\title{
Drift Rejection Differential Frontend for Single Plate Capacitive Sensors
}

\author{
Mihai T. Lazarescu ${ }^{1 *}$ \\ ${ }^{1}$ Department of Electronics and Telecommunications, Politecnico di Torino, 10129 Torino, Italy \\ * Senior Member, IEEE
}

\begin{abstract}
Using capacitive sensors at long ranges (10-20x their plate diameter) for long term environmental sensing can be limited by slow but significant measurement drifts that can often far exceed the small capacitance variations of interest, which can be around $0.01 \%$ or less. We propose a differential capacitance measurement method that rejects the quasi-constant drift currents for single plate capacitive sensors by averaging the absolute slope values of adjacent chargedischarge voltage ramps of the plate capacitance, under constant current. Compared analytically and in simulations with period modulation techniques using astable multivibrators, our method shows much better rejection of drifts due to quasiconstant charge migration and improved random noise attenuation, while preserving the measurement sensitivity. We also provide an implementation that avoids errors caused by some types of ramp distortions and improves noise reduction.
\end{abstract}

Index Terms-Capacitive sensor, differential measurement, drift rejection, noise reduction, oversampling, environmental monitoring.

\section{INTRODUCTION}

Capacitive sensors find many applications, e.g., for human-machine interfaces [1], measurement [2], or sensing [3]. They can cover from very large surfaces [4] down to nanostructures [5], can be used for many sensing needs from gas detectors [6], to tomography [7] and defectoscopy [8], with low cost and low power consumption.

However, capacitance measurement can be affected by multiple environmental factors, which typically limit their use to ranges at most comparable to plate diameter [9], where their measurement field can be effectively shielded using various techniques [10]. But longer sensing ranges, e.g., at 10-20× the plate diameter, are needed for applications requiring inconspicuous indoor person localization [11] or identification [12] using small low power and low cost sensors, for home automation [13] or assisted living [14]. At longer ranges, the capacitance measurement field is often exposed to environmental noise, which can significantly reduce the sensor range and stability.

Drift from various sources often affects capacitive sensor measurements for long term monitoring, and especially when they are used for long range sensing, where capacitance changes can be very small, around $0.01 \%$ (100 ppm) or lower [11], [15]. Measurement drift can be due to changes of the sensor capacitance, e.g., because of variable relative humidity of the air, which can be separately measured and compensated. Another drift cause can be due to environmental influences on the parameters of the capacitance measurement, but which do not correspond to changes in the actual sensor capacitance.

Relaxation oscillators are often used to measure the sensor capacitance. It modulates the period of their oscillation and can be digitalized using a microcontroller counter clocked by an accurate time references. The interface presented in [16] uses self-calibration and several trade-offs to significantly reduce the power consumption of the interface to be suitable for long term monitoring using wireless sensor networks. The authors introduce in [17] a Schmitt trigger with thresholds easy to adjust, and which they demonstrate in an astable multivibrator circuit for capacitance measurement over a very wide range. In [18], the authors propose a period modulation interface for

Corresponding author: M. T. Lazarescu (e-mail: mihai.lazarescu@polito.it). sub-nanometer displacement measurement using capacitive sensors, using chopping, self-calibration, and active shielding to reduce errors.

In our previous work [19], we used a simple period modulation interface based on a 555 timer [20] to acquire capacitive sensor measurements for person localization using machine learning with good results. However, sensor measurements had slow but significant drifts, which depended both on sensor location and its handling before the experiments, attributable to static charge transfer.

We examine sensor drift caused by charge migration for single plate long range capacitive sensors and propose a capacitance measurement method that rejects it while preserving the measurement sensitivity.

\section{SENSOR DRIFT PROBLEM DEFINITION}

Capacitance $C$ of an object is defined as the ratio between charge variation, $\Delta Q$, and potential (voltage to a reference) variation, $\Delta V_{C}$

$$
C=\frac{\Delta Q}{\Delta V_{C}} .
$$

While capacitance depends only on electric and dielectric properties, and system geometry, the measurement methods can be susceptible to environmental factors with no bearing on capacitance value, such as electric and electromagnetic fields.

Fig. 1 shows a typical period modulation (astable multivibrator) interface based on (1), which can also be used when only one sensor plate is connected, e.g., for capacitive sensors operating in load mode [19]. Plate charge is changed in the time $\Delta t$ by a constant current $I$

$$
\Delta Q=I \Delta t
$$

and replacing (2) in (1) with $C=C_{\text {plate }}$ constant, $V_{C}$ changes as

$$
\Delta V_{C}=\frac{I}{C_{\text {plate }}} \Delta t .
$$

I can be generated with a current source controlled by the output $v$ of a hysteresis comparator, with low $\left(V_{\mathrm{TL}}\right)$ and high $\left(V_{\mathrm{TH}}\right)$ thresholds. $v$ swings between $\pm 1 \mathrm{~V}$ as the plate voltage $V_{C}$ reaches the thresholds, changing the sign of $I$ such way to satisfy $V_{\mathrm{TL}} \leq V_{C} \leq V_{\mathrm{TH}}$ [see the dashed plot in Fig. 2(b)]. Assuming $C_{\text {plate }}$ constant and no noise, we obtain from (3) equal charge and discharge times, respectively

$$
C_{\text {plate }} \frac{V_{\mathrm{TH}}-V_{\mathrm{TL}}}{I}=C_{\text {plate }} \frac{V_{\mathrm{TL}}-V_{\mathrm{TH}}}{-I}=\frac{T_{N}}{2} \text {. }
$$




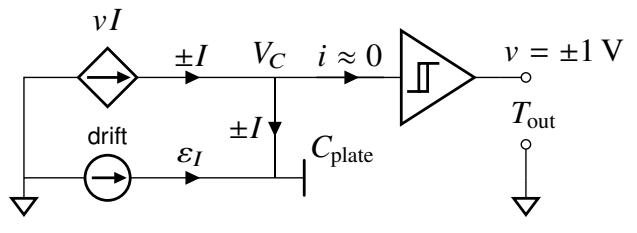

Fig. 1. Capacitance-to-period conversion circuit with drift current, $\varepsilon_{I}$

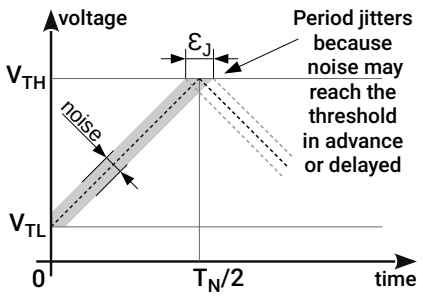

(a)

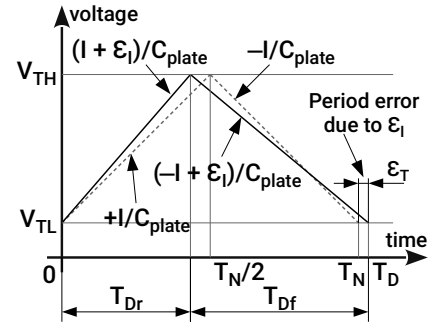

(b)
Fig. 2. Period with jitter due to (a) noise and errors due to (b) drift, $\varepsilon_{I}$.

We can calculate $C_{\text {plate }}$ if we know the oscillation period, $T_{N}$

$$
C_{\text {plate }}=\frac{I}{2\left(V_{\mathrm{TH}}-V_{\mathrm{TL}}\right)} T_{N}
$$

and the sensitivity of its output frequency to $C_{\text {plate }}$ variations

$$
\frac{\partial f_{N}}{\partial C_{\text {plate }}}=-\frac{1}{2\left(V_{\mathrm{TH}}-V_{\mathrm{TL}}\right)} \cdot \frac{I}{C_{\text {plate }}^{2}}, \quad \text { with } f_{N}=\frac{1}{T_{N}} .
$$

However, the environmental noise can change $T_{N}$, hence introduce errors in the measured $C_{\text {plate }}$ value, in two major ways.

First, high-pitch environmental noise can change randomly the $V_{C}$ ramp amplitude [e.g., within the gray area in Fig. 2(a)], leading to an $\varepsilon_{J}$ jitter of the time when the ramp actually reaches the thresholds. $\varepsilon_{J}$ affects the stability of the oscillation period $T_{N}$, hence from (5) the measured $C_{\text {plate }}$. While the jitter noise reduces sensor sensitivity, it eventually averages close to zero for long measurement times.

Second, the charge migration in the sensor plate due to environmental electric field variations slower than the oscillation period $T_{N}$ acts as a current source $\varepsilon_{I}$ (assumed constant to simplify calculations). It adds algebraically to the controlled oscillator current $\pm I$ [see Fig. 2(b)], changes the rising, $T_{\mathrm{Dr}}$, and falling, $T_{\mathrm{Df}}$, ramp durations in (4)

$$
T_{\mathrm{Dr}}=C_{\text {plate }} \frac{V_{\mathrm{TH}}-V_{\mathrm{TL}}}{I+\varepsilon_{I}}, \quad T_{\mathrm{Df}}=C_{\text {plate }} \frac{V_{\mathrm{TL}}-V_{\mathrm{TH}}}{-I+\varepsilon_{I}}
$$

as well as the oscillation period, $T_{D}$

$$
T_{D}=T_{\mathrm{Dr}}+T_{\mathrm{Df}}=\frac{2 C_{\mathrm{plate}}\left(V_{\mathrm{TH}}-V_{\mathrm{TL}}\right) I}{I^{2}-\varepsilon_{I}^{2}} .
$$

From (5) and (8), we calculate the relative measurement error $T_{e_{r}}$

$$
T_{e_{r}}=\frac{T_{D}-T_{N}}{T_{N}}=\frac{I^{2}}{I^{2}-\varepsilon_{I}^{2}}-1=\frac{\varepsilon_{I}^{2}}{I^{2}-\varepsilon_{I}^{2}} .
$$

$\varepsilon_{I}$ systematically increases the oscillation period (sensor output), reducing the sensor stability especially at low level signals, i.e. at the long sensing ranges important for monitoring applications [11].

\section{PROPOSED DRIFT REJECTION DESCRIPTION}

We aim to reduce the drift of capacitance measurements based on (1) from externally-induced plate charge migrations (9).

We use the slope modulation measurement circuit in Fig. 3. As in

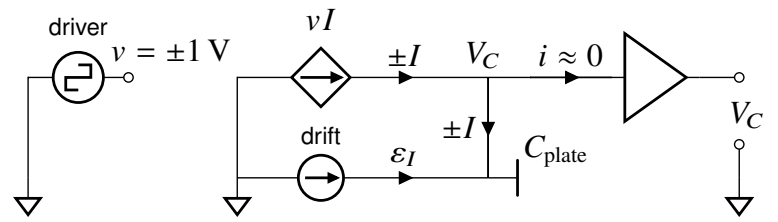

Fig. 3. Capacitance-to-slope conversion circuit with drift current, $\varepsilon_{I}$

the circuit in Fig. 1, $C_{\text {plate }}$ charge is changed by a current generated by a source controlled with a voltage $v$. Unlike the case shown in Fig. 2(a), $v$ timing is fixed, $T_{m}$ (see Fig. 4), regardless of $V_{C}$ noise.

From (3), we get the $V_{C}$ ramp slope $S$ inversely proportional to $C_{\text {plate }}$

$$
S=\frac{\Delta V_{C}}{\Delta t}=\frac{I}{C_{\text {plate }}} .
$$

Assuming constant charge migration, $\varepsilon_{I}$, the rising $\left(S_{r}\right)$ and falling $\left(S_{f}\right)$ slopes are

$$
S_{r}=\frac{I+\varepsilon_{I}}{C_{\text {plate }}}, \quad S_{f}=\frac{-I+\varepsilon_{I}}{C_{\text {plate }}}
$$

the average $S_{a}$ of slope modulus is

$$
S_{a}=\frac{\left|S_{r}\right|+\left|S_{f}\right|}{2}=\frac{1}{2}\left(\frac{I+\varepsilon_{I}}{C_{\text {plate }}}-\frac{-I+\varepsilon_{I}}{C_{\text {plate }}}\right)=\frac{I}{C_{\text {plate }}}
$$

and the circuit sensitivity to $C_{\text {plate }}$ variations is

$$
\frac{\partial S_{a}}{\partial C_{\text {plate }}}=-\frac{I}{C_{\text {plate }}^{2}} .
$$

We see from (12) that the system rejects quasi-constant charge migration currents, $\varepsilon_{I}$, which is useful to reject drifts due to slow environmental electric field changes during long term monitoring.

Note also that capacitance measurement in this method is based on the average ramp slope, which is less sensitive to noise than detecting the specific instants when the ramps touch the voltage thresholds.

\section{EXPERIMENTAL RESULTS}

We discuss the implementation that we used to test the proposed method, described in Section III. Then, we discuss several simulation results and compare the drift rejection and sensitivity of the proposed method and of the reference astable multivibrator reference technique.

Note that we report the raw interface output because most environmental sensing applications use relative capacitance variations.

\section{A. System implementation}

Knowing the circuit constant $I, C_{\text {plate }}$ can be calculated using (12) if we measure two adjacent rising and falling slopes of $V_{C}$

$$
C_{\text {plate }}=\frac{2 I}{S_{r}-S_{f}} \text {. }
$$

Fig. 4 shows the main elements that we use to measure the slopes. $V_{C}$ (thin blue triangular wave) is plotted from an LTspice [21] simulation of the circuit shown in Fig. 3. We use an Analog to Digital Converter (ADC) to acquire $2 N_{R} V_{C}$ samples over a full period, $T_{m}$. We calculate with the samples the $V_{C}$ ramp slopes, then $C_{\text {plate }}$ with (14). The ADC must be synchronized with the start of the $V_{C}$ period, which can be easily achieved, e.g., if both the $V_{C}$ driver, $v$, and the $\mathrm{ADC}$ are driven by the same microcontroller.

We need two points to calculate the slope of each $V_{C}$ ramp, see $A_{1}$ and $A_{2}$ in Fig. 4 for $S_{f}$, and $A_{3}$ and $A_{4}$ for $S_{r}$. To improve 


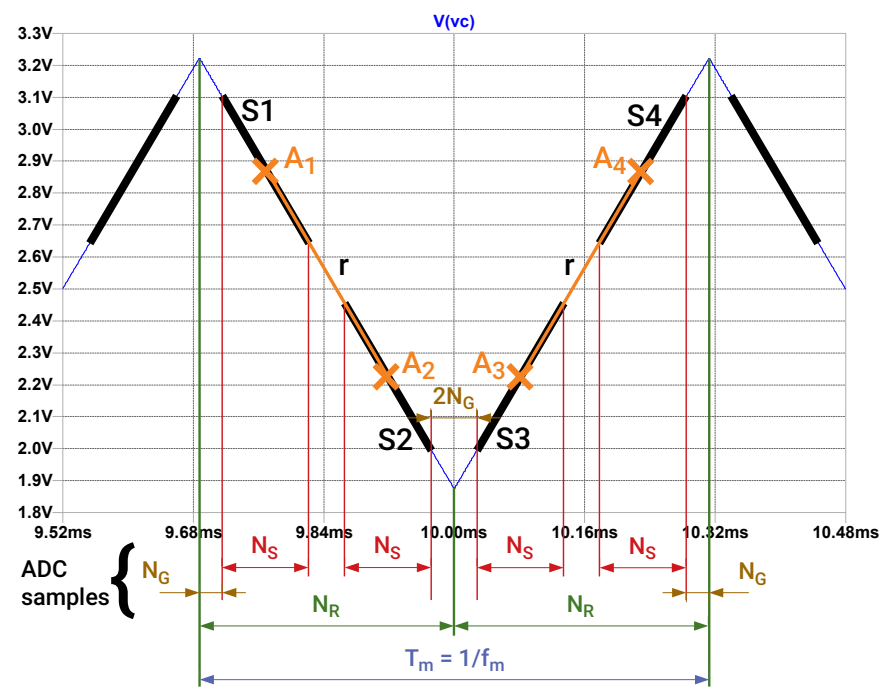

Fig. 4. Double slope measurement timing on Spice simulation

the measurement accuracy, the points must be 1) well spaced (close to ramp ends, but not too close, see below) and 2) calculated by averaging multiple sequential ADC samples over a ramp segment (see segments $S_{1-4}$ for points $A_{1-4}$, respectively, in Fig. 4), to reduce the high-pitch uncorrelated noise. As mentioned, the segments $S_{1-4}$ must avoid ramp ends, which can be distorted by, e.g., clipping due to voltage range limits of circuit components or by anti-aliasing filters (see the discarded $N_{G}$ samples in Fig. 4).

Voltages $V_{A_{i}}$ of points $A_{i}, i \in\{1,2,3,4\}$ are calculated using an $N_{S}$-sample ramp segment, $S_{i}$. We set $N_{S}$ a non-zero power of four to lower discretization noise through oversampling and decimation [22]

$$
V_{A_{i}}=\frac{1}{\frac{N_{S}}{2}} \sum_{j=1}^{N_{S}} V_{S_{i, j}}, \quad i \in\{1,2,3,4\} \quad \text { if } N_{S}=4^{n}, n \in \mathbb{N}^{+}
$$

$V_{S_{i}}$ being the voltage of ramp samples in segment $S_{i}$. The time $T_{A_{1,2}}$ between $A_{1}$ and $A_{2}$, equal to the time $T_{A_{3,4}}$ between $A_{3}$ and $A_{4}$, is

$$
T_{A_{1,2}}=T_{A_{3,4}}=\frac{T_{m}}{2 N_{R}}\left[N_{R}-2\left(N_{G}+\frac{N_{S}}{2}\right)\right]
$$

Substituting (16) in (10), the ramp slopes are

$$
S_{r}=\frac{2 N_{R}\left(V_{A_{4}}-V_{A_{3}}\right)}{T_{m}\left(N_{R}-2 N_{G}-N_{S}\right)}, \quad S_{f}=\frac{2 N_{R}\left(V_{A_{2}}-V_{A_{1}}\right)}{T_{m}\left(N_{R}-2 N_{G}-N_{S}\right)}
$$

and substituting (17) in (14), we obtain $C_{\text {plate }}$

$$
C_{\text {plate }}=\frac{I T_{m}}{V_{A_{4}}-V_{A_{3}}-\left(V_{A_{2}}-V_{A_{1}}\right)} \cdot \frac{N_{R}-2 N_{G}-N_{S}}{N_{R}} .
$$

The system design must determine a driver $(v)$ frequency, $f_{m}=1 / T_{m}$ (see Fig. 4), at least twice the highest frequency in the sensing application to satisfy the Nyquist-Shannon sampling theorem [23]. The ADC should acquire an even number, $2 N_{R}$, of samples in each $f_{m}$ period, hence $N_{R}$ samples per ramp. In $N_{R}$ should fit: two $N_{S}$-sample segments, with $N_{S}$ a non-zero power of four, $2 N_{G}$ samples that are ignored at each ramp end to skip potential local ramp distortions, and $r$ samples to round up the sum to $N_{R}$, placed between the two $N_{S}$-sample segments (see Fig. 4)

$$
N_{R}=2 N_{S}+2 N_{G}+r .
$$

$N_{G}$ should be kept small but sufficiently large to cover circuitspecific non-linearities, e.g., due to the anti-alias filters that can be inserted between the $V_{C}$ output in Fig. 3 and the ADC. In Fig. 5,

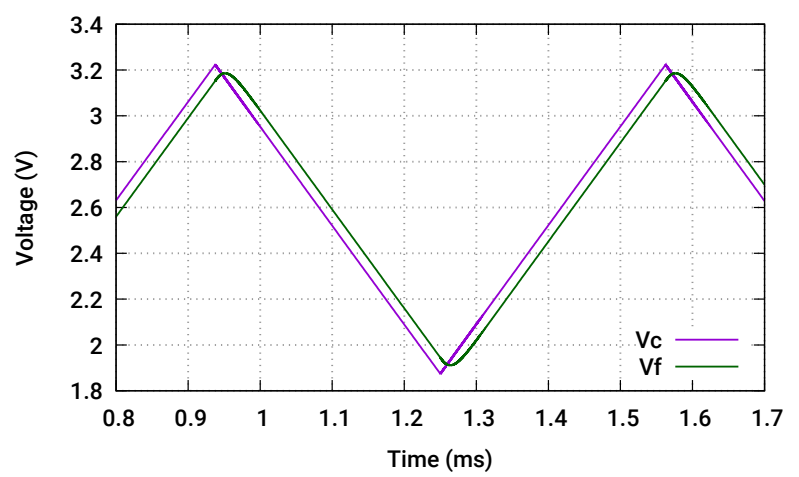

Fig. 5. Anti-alias low-pass filter delays ramps and distorts their ends.

we show an example of phase shift and ramp-end distortions from a $20 \mathrm{kHz}$ second order low-pass filter $\left(V_{f}\right)$ on a $1.6 \mathrm{kHz} V_{C} . N_{G}$ should be dimensioned to skip the non-linear ramp ends of $V_{f}$ and ADC sampling should be delayed to match the filter phase shift.

Note that unlike the circuit in Fig 1 , where $V_{C}$ is constrained between the $V_{\mathrm{TL}}$ and $V_{\mathrm{TH}}$ comparator thresholds, the circuit in Fig. 3 does not control the $V_{C}$ absolute value. Small circuit asymmetries or environmental influences may accumulate over time and eventually push $V_{C}$ in the non-linear operation range of circuit components, which may lead to distortions, e.g., clipping. Additionally, for a given $C_{\text {plate }}$, the current $I$ and the driver period $T_{m}$ should be dimensioned to keep the amplitude of $V_{C}$ lower than the linear range of the circuit.

\section{B. Drift rejection}

Circuit parameters should be designed to satisfy the application requirements and the design criteria and conditions in Section IV-A.

In absence of well-defined application requirements, we use for our testing an LTspice simulation of the slope modulation measurement circuit shown in Fig. 3 with the following parameters: $C_{\text {plate }}=25 \mathrm{pF}$ (from our previous experiments [19]), $I \approx 112.5 \mathrm{nA}$, ADC sampling frequency $2 \mathrm{Msample} / \mathrm{s}, f_{m}=1.6 \mathrm{kHz}$ (hence $N_{R}=625$ samples) and $N_{S}=4^{4}=256$ samples. Although we do not expect ramp distortions, we set the largest possible $N_{G}=56$ samples (for worstcase measurement accuracy), hence the segments are spaced by $r=1$ sample, calculated with (19). With these settings, $V_{C}$ swings about $1.41 \mathrm{~V}$, compatible with typical circuit supplies, e.g., $3.3 \mathrm{~V}$.

For comparison, we simulate the period modulation (astable multivibrator) measurement circuit in Fig. 1 in similar conditions: $C_{\text {plate }}=25 \mathrm{pF}, I=112.5 \mathrm{nA}, T_{N} \approx 619.6 \mu$ s (or frequency around $1614 \mathrm{~Hz}$ ), and $V_{\mathrm{TH}}-V_{\mathrm{TL}} \approx 1.39 \mathrm{~V}$.

In each simulation, we model the charge migration currents in both directions, $\varepsilon_{I}= \pm 1 \mathrm{nA}$ and check their effects on circuit output. We also change $C_{\text {plate }}$ value to compare the sensitivity of the circuits.

Fig. 6(a) shows the oscillation frequency output of the period modulation measurement circuit in Fig. 1. We show the frequency output instead of the period to facilitate the comparison of results between methods, because $C_{\text {plate }}$ is inversely proportional to both the oscillation frequency (5) and the average slope (12).

When $\varepsilon_{I}=0 \mathrm{nA}$, before $10 \mathrm{~ms}$ and after $30 \mathrm{~ms}$, the output period is stable around $1614.52 \mathrm{~Hz}$. However, as shown by (9), the circuit is equally influenced by migration currents $\varepsilon_{I}$ of either direction, $+1 \mathrm{nA}$ between $10 \mathrm{~ms}$ and $20 \mathrm{~ms}$, and $-1 \mathrm{nA}$ between $20 \mathrm{~ms}$ and $30 \mathrm{~ms}$, both lowering its output frequency around $1614.40 \mathrm{~Hz}$. 


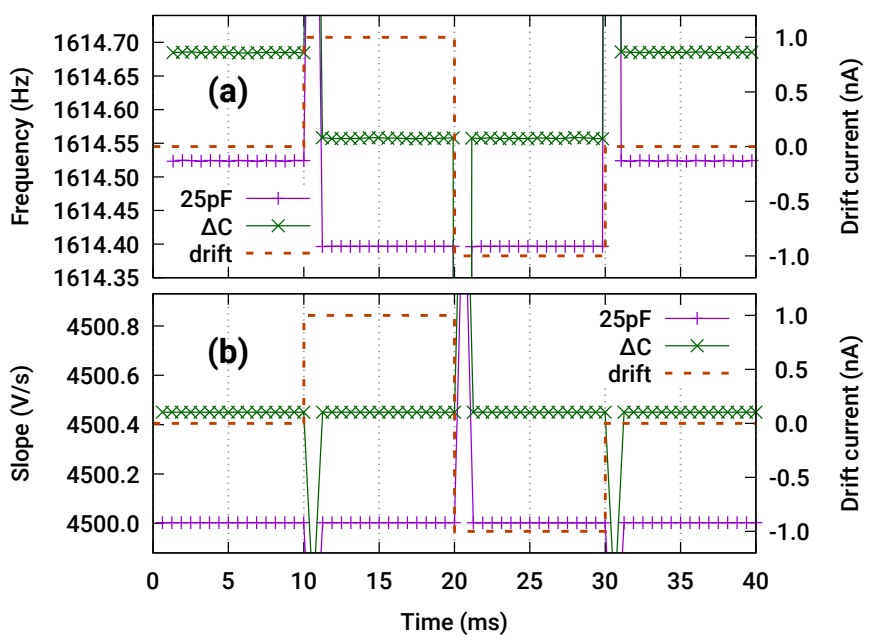

Fig. 6. Output sensitivity to charge migration (drift current) $\varepsilon_{I}$ and to plate capacitance change $\Delta C=100 \mathrm{ppm}$ below the nominal $25 \mathrm{pF}$ for (a) period modulation and (b) proposed slope modulation method

To test the sensitivity to $C_{\text {plate }}$ variations, we change $C_{\text {plate }}$ and simulate again the circuit response. We are mostly interested in longrange measurements, where $C_{\text {plate }}$ can vary around $100 \mathrm{ppm} \mathrm{[19].} \mathrm{So}$ we lower $C_{\text {plate }}$ by $100 \mathrm{ppm}$ [or $2.5 \mathrm{fF}, \Delta C$ in Fig. 6(a)], and the output frequency increases, as of (6).

Fig. 6(b) shows the output of the proposed slope modulation measurement circuit in Fig. 3 in the same operating conditions. As shown in (12), its output remains stable, around $4500 \mathrm{~V} \mathrm{~s}^{-1}$, for all tested values of the charge migration current $\varepsilon_{I}$. Its output increases when we lower $C_{\text {plate }}$ by the same $100 \mathrm{ppm}(25 \mathrm{fF})$, as of (13). Compared with (6), this circuit is more sensitive by a ratio

$$
\frac{\partial S_{a}}{\partial C_{\text {plate }}}\left(\frac{\partial f_{N}}{\partial C_{\text {plate }}}\right)^{-1}=2\left(V_{\mathrm{TH}}-V_{\mathrm{TL}}\right)
$$

or $2 \cdot 1.39 \mathrm{~V}=2.78 \mathrm{~V}$ in this test, within $5 \%$ of the ratio between the output changes of the two circuits (simulation and measurement errors)

$$
\frac{4500.45 \mathrm{~V} \mathrm{~s}^{-1}-4500.00 \mathrm{~V} \mathrm{~s}^{-1}}{1614.69 \mathrm{~Hz}-1614.52 \mathrm{~Hz}} .=\frac{0.45 \mathrm{~V} \mathrm{~s}^{-1}}{0.17 \mathrm{~Hz}} \approx 2.65 \mathrm{~V}
$$

Note that we clipped for readability the output spikes corresponding to sudden $\varepsilon_{I}$ changes because we focus on long term drifts with quasi-constant $\varepsilon_{I}$ and because in our circuit they are significantly attenuated by the anti-alias filter discussed in Section IV-A.

\section{CONCLUSIONS}

Differential measurement of adjacent capacitance charge-discharge ramp slopes effectively rejects quasi-constant drift currents which affect the stability of capacitive sensors for long-time environmental monitoring. Moreover, each measurement is based on averaging several data points, which is less susceptible to noise. The technique can be used for single plate sensors and does not have special requirements for the sensing element. Compared with measurement methods based on detection of threshold crossing, it shows very good drift current rejection while preserving the measurement sensitivity.

\section{REFERENCES}

[1] T. Grosse-Puppendahl, C. Holz, G. Cohn, R. Wimmer, O. Bechtold, S. Hodges, M. S. Reynolds, and J. R. Smith, "Finding common ground: A survey of capacitive sensing in human-computer interaction," in Proceedings of the 2017 CHI Conference on Human Factors in Computing Systems. ACM, 2017, pp. 3293-3315.

[2] F. Reverter, X. Li, and G. C. Meijer, "Liquid-level measurement system based on a remote grounded capacitive sensor," Sensors and Actuators A: Physical, vol. 138, no. 1, pp. 1-8, 2007.

[3] Y. Liu, J. Deng, Y. Ye, C. Han, Z. Hou, and Z. Duan, "Capacitive sensor for vehicle obstacle detection, especially for pedestrian detection," Microsystem Technologies, Oct 2019. [Online]. Available: https://doi.org/10.1007/s00542-019-04647-1

[4] S. Laflamme, M. Kollosche, J. J. Connor, and G. Kofod, "Soft capacitive sensor for structural health monitoring of large-scale systems," Structural Control and Health Monitoring, vol. 19, no. 1, pp. 70-81, 2012.

[5] Y. Zhang, R. Howver, B. Gogoi, and N. Yazdi, "A high-sensitive ultra-thin MEMS capacitive pressure sensor," in 2011 16th International Solid-State Sensors, Actuators and Microsystems Conference. IEEE, 2011, pp. 112-115.

[6] H.-E. Endres, R. Hartinger, M. Schwaiger, G. Gmelch, and M. Roth, "A capacitive $\mathrm{CO} 2$ sensor system with suppression of the humidity interference," Sensors and Actuators B: Chemical, vol. 57, no. 1-3, pp. 83-87, 1999.

[7] S. Wöckel, U. Hempel, and J. Auge, "Acousto-capacitive tomography of liquid multiphase systems," Sensors and Actuators A: Physical, vol. 172, no. 1, pp. 322-329, 2011

[8] A. Kakimoto, "Detection of surface defects on steel ball bearings in production process using a capacitive sensor," Measurement, vol. 17, no. 1, pp. 51-57, 1996

[9] J. Boehm, R. Gerber, and N. Kiley, "Sensors for magnetic bearings," IEEE Trans. Magn., vol. 29, no. 6, pp. 2962-2964, 1993.

[10] F. Reverter, X. Li, and G. C. Meijer, "Stability and accuracy of active shielding for grounded capacitive sensors," Measurement Science and Technology, vol. 17. no. 11 , p. $2884,2006$.

[11] O. B. Tariq, M. T. Lazarescu, J. Iqbal, and L. Lavagno, "Performance of machine learning classifiers for indoor person localization with capacitive sensors," IEEE Access, vol. 5, pp. 12913-12926, 2017.

[12] J. Iqbal, M. T. Lazarescu, O. B. Tariq, A. Arif, and L. Lavagno, "Capacitive Sensor for Tagless Remote Human Identification Using Body Frequency Absorption Signatures," IEEE Trans. Instrum. Meas., vol. 67, no. 4, pp. 789-797, 2018.

[13] A. Braun, S. Rus, and M. Majewski, "Invisible Human Sensing in Smart Living Environments Using Capacitive Sensors," in Ambient Assisted Living. Springer, 2017, pp. 43-53.

[14] S. A. Lowe and G. ÓLaighin, "Monitoring human health behaviour in one's living environment: a technological review," Medical engineering \& physics, vol. 36, no. 2, pp. 147-168, 2014.

[15] D. Kang, W. Lee, and W. Moon, "A technique for drift compensation of an areavarying capacitive displacement sensor for nano-metrology," Procedia Engineering, vol. 5, pp. 412-415, 2010.

[16] Z. Tan, S. H. Shalmany, G. C. Meijer, and M. A. Pertijs, "An energy-efficient 15-bit capacitive-sensor interface based on period modulation," IEEE J. Solid-State Circuits, vol. 47, no. 7, pp. 1703-1711, 2012.

[17] A. De Marcellis, G. Ferri, and P. Mantenuto, "A CCII-based non-inverting Schmitt trigger and its application as astable multivibrator for capacitive sensor interfacing," Int. J. Circ. Theor. Appl., vol. 45, no. 8, pp. 1060-1076, 2017.

[18] A. Ahmadpour Bijargah, A. Heidary, P. Torkzadeh, and S. Nihtianov, "An accurate and power-efficient period-modulator-based interface for grounded capacitive sensors," International Journal of Circuit Theory and Applications, 2019.

[19] A. Ramezani Akhmareh, M. Lazarescu, O. Bin Tariq, and L. Lavagno, "A tagless indoor localization system based on capacitive sensing technology," Sensors, vol. 16, no. 9, p. 1448, 2016.

[20] H. R. Camenzind and R. B. Kash, “A low-voltage IC timer,” IEEE J. Solid-State Circuits, vol. 13, no. 6, pp. 847-852, 1978.

[21] “LTspice I Design Center I Analog Devices." [Online]. Available: https://www. analog.com/en/design-center/design-tools-and-calculators/ltspice-simulator.html

[22] P. Harpe, E. Cantatore, and A. van Roermund, "An oversampled 12/14b SAR ADC with noise reduction and linearity enhancements achieving up to 79.1 dB SNDR," in 2014 IEEE International Solid-State Circuits Conference Digest of Technical Papers (ISSCC). IEEE, 2014, pp. 194-195.

[23] C. E. Shannon, "Communication in the presence of noise," Proceedings of the IRE, vol. 37, no. 1, pp. 10-21, 1949. 